function and valvulopathy, with two-dimensional, Doppler, tissue Doppler, and speckle tracking echocardiography. We included for meta-analysis all controlled studies including ax-SpA without previous cardiovascular disease. Data were pooled using appropriate random or fixed effects model.

Results: Literature search retrieved of 186 abstracts. A total of 31 papers were included in the systematic review and 27 papers were analyzed in the meta-analysis (1,494 ax-SpA patients and 1,091 healthy controls). Studies displayed cross-sectional design and included ax-SpA without prevalent cardiovascular disease.

Ax-SpA was defined according to the modified New York criteria (24 studies) followed or the ASAS criteria (2 studies). HLA B27+ positivity ranged from 51 to $100 \%$, mean age ranged from 26.7 to 55.7 years, disease duration ranged from 3.2 to 23.3 years and mean BASDAl ranged from 1.24 to 5.6 .

Patients with ax-SpA displayed a lower diastolic function with a lower E/A ratio, a higher deceleration time, a higher isovolumetric relaxation time and a lower systolic function with a lower ejection fraction (figure 1). Left-ventricular end diastolic and systolic diameters were higher in ax-SpA patients with respectively mean difference $0.55 \mathrm{~mm}[\mathrm{Cl} 95 \% ; 0.19,0.91]$ and $0.79 \mathrm{~mm}$ [Cl95\%; 0.40, 1.17]. We did not find any difference for left and posterior ventricular thickness, left atrial dimension, and left ventricular mass index.

A
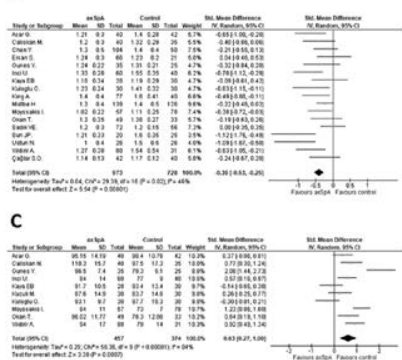

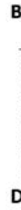

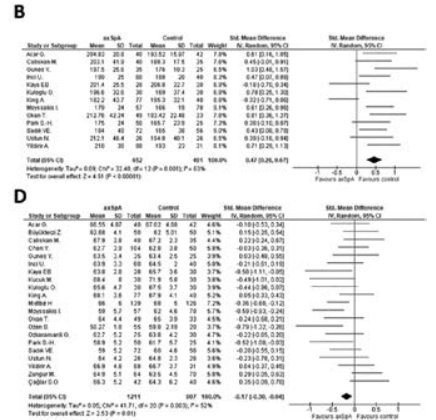

Figure 1. Systolic and diastolic dysfunction is slightly altered in ax-SpA patients compared to healthy individualsDiastolic dysfunction was assessed by $(A) E / A$ ratio $(\mathrm{m} / \mathrm{s}),(B)$ deceleration time (ms), (C) Isovolumetric relaxation time (ms) and (D) systolic function was assessed by ejection fraction (\%).

A total of 15 articles reported prevalence of valvulopathy in ax-SpA. Prevalence of mitral regurgitation and aortic regurgitation were similar in ax-SpA patients and healthy individuals: $\mathrm{OR}=1.13[\mathrm{Cl} 95 \% 0.76,1.68]$ and $\mathrm{OR}=1.18$ [CI95\% 0.68, 2.04]. Conclusion: Prevalence of valvulopathy was similar in ax-SpA and healthy individuals. Diastolic and systolic function seems to be slightly altered in ax-SpA compared to healthy controls. However, this difference is unlikely clinically relevant. Usefulness of systematic echography remains to be determined in future longitudinal studies.

Disclosure of Interests: Fanny Adeline: None declared, Xavier Romand Consultant of: Xavier ROMAND has received honorarium fees from Abbvie, Mickael Dalecky Consultant of: Mickael DALECKY has received honorarium fees from Abbvie, Arnaud Pfimlin Consultant of: Arnaud PFIMLIN has received honorarium fees from Abbvie, Daniel Wendling: None declared, Philippe Gaudin Speakers bureau: Lilly, Pascal Claudepierre Speakers bureau: Janssen, Novartis, Lilly, Maxime Dougados Grant/research support from: AbbVie, Eli Lilly, Merck, Novartis, Pfizer and UCB Pharma, Consultant of: AbbVie, Eli Lilly, Merck, Novartis, Pfizer and UCB Pharma, Speakers bureau: AbbVie, Eli Lilly, Merck, Novartis, Pfizer and UCB Pharma, Athan Baillet Consultant of: Athan BAILLET has received honorarium fees from Abbvie for his participation as the coordinator of the systematic literature review

DOI: 10.1136/annrheumdis-2020-eular.5223

\begin{tabular}{|l|l|}
\hline AB0666 & A COMPARISON OF CLINICAL FEATURES AND \\
PREDICTORS OF TREATMENT RESPONSE IN \\
SPONDYLOARTHRITIS PATIENTS IN THE MIDDLE \\
EAST: A CROSS-SECTIONAL MULTINATIONAL \\
STUDY
\end{tabular}

J. Al-Saleh ${ }^{1}$, M. P. Abi Saab ${ }^{2}$, A. Negm ${ }^{1}$, F. Balushi ${ }^{3}$, R. Namas ${ }^{4}$, N. Ziade ${ }^{5}$. ${ }^{1}$ Dubai Hospital, Rheumatology Department, Dubai, United Arab Emirates; ${ }^{2} \mathrm{Al}$ Ahli Hospital, Qatar, Qatar; ${ }^{3}$ Royal Hospital, Muscat, Oman; ${ }^{4}$ Cleveland Clinic, Abu Dhabi, United Arab Emirates; ${ }^{5}$ Hotel-Dieu de France Hospital and SaintJoseph University Beirut, Beirut, Lebanon

Background: Spondylarthritis it is a chronic inflammatory disease with heterogenous clinical features. Its prevalence ranges between $0.2 \%-2 \%$. Over the years biological therapy has improved work productivity and activity impairment in people with SpA. Unlike in rheumatoid arthritis, the concept of treat-to-target is still debatable among rheumatologist. However, there is a consensus that treatment in patient with SpA should be personalized. There are several challenges in the Middle East that might affect providing personalized medicine to patients with $\mathrm{SpA}$ in this region.

Objectives: The of objective of the study is to explore factors that interfere with achieving clinical targets in patients with SpA clinical practice in the Middle East. Methods: We conducted a cross-sectional, multicentre study to explore the factors that interfere with achieving clinical targets in SpA patients from four countries in the Middle East (Lebanon, Oman, Qatar, and the United Arab Emirates). A total of 404 patients who attended participating centers from January 2019 to June 2019 and who met the ASAS 2010 classification criteria for axial and peripheral SpA; and were at least 18 years of age were enrolled in the study. We excluded patients with peripheral arthritis only. We extracted demographics, clinical data, and conducted patients survey. We used Compliance Questionnaire for Rheumatology (CQR) is a self-reported adherence measure created specifically for and validated in rheumatic diseases.

Demographic data and disease and treatment characteristics were described as median and the 25th-75th interquartile range (IQR). Multiple regression analysis was used to investigate the impact of different factors on ASDAS-CRP in patients with SpA. Statistical analysis was performed using Minitab version 18.1 software. Results: A total of 404 patients initially enrolled in the study, we excluded 95 patients as they had peripheral involvement only. We analysed the data of 309 patients with axial only or axial and peripheral SpA. There median age was 43 years and $53.7 \%$ were females. The median disease duration was six years. At the time of the study, $72.1 \%$ patients were within the arbitrary clinical target of ASDAS $<2$.1. Detail description of the studied population and subgroups outlined in table 1. Enthesitis (OR: 2.9; $P$ value: 0.004 ), Psoriasis (OR: $2.74 ; P$ value: 0.007 ), low compliance score (OR: -4.36 ; P value: < 0.0001) and HLA B27 (OR: 2.12; P value: $<0.04$ ) were independent predictors of a higher ASDAS -CRP.

Conclusion: Enthesitis, psoriasis, noncompliance, and HLA B27 were independent predictors for ASDAS in our cohort.

Table 1. Demographic and clinical characteristics of all patients and for achiever and non-achievers

\begin{tabular}{lccc}
\hline & $\begin{array}{c}\text { All patients } \\
\text { Variables }\end{array}$ & $\begin{array}{c}\text { Achiever } \\
\mathbf{( 3 0 9 )}\end{array}$ & $\begin{array}{c}\text { Non-achieves } \\
\mathbf{( 2 6 )}\end{array}$ \\
\hline Age, Median, (IQR) yrs & $43,(36-51)$ & $43,(35-51.5)$ & $42,(37-51)$ \\
Female \% & $53.7 \%$ & $54.2 \%$ & $51.9 \%$ \\
Disease Duration, Median, (IQR)yrs & $6,(3-9)$ & $6,(2-8)$ & $7.5,(3-10)$ \\
Patient has medical insurance/ Medical coverage & $94.5 \%$ & $95.0 \%$ & $93.1 \%$ \\
Smoking & $13.9 \%$ & $12.1 \%$ & $18.6 \%$ \\
ASDAS-CRP, Median (IQR) & $1.56,(1.24-2.1)$ & $1.56,(1.07-1.6)$ & 2.75 \\
& & & $(2.36-3.3)$ \\
Arthritis & $40.1 \%$ & $36.3 \%$ & $48.1 \%$ \\
Dactylitis & $13.6 \%$ & $11.3 \%$ & $18.50 \%$ \\
Enthesitis & $29.1 \%$ & $22.4 \%$ & $43.2 \%$ \\
Family history of SPA & $18.4 \%$ & $14.3 \%$ & $25.9 \%$ \\
Good response to NSAIDs & $21.7 \%$ & $18.8 \%$ & $22.2 \%$ \\
HLA B27 & $30.0 \%$ & $25.6 \%$ & $40.0 \%$ \\
Inflammatory bowel disease & $7.1 \%$ & $7.3 \%$ & $6.20 \%$ \\
Inflammatory low back pain & $68.6 \%$ & $68.1 \%$ & $69.8 \%$ \\
Onycholysis & $10.9 \%$ & $10.7 \%$ & $11.1 \%$ \\
Psoriasis & $39.4 \%$ & $25.1 \%$ & $40.7 \%$ \\
Sacroilitis (Radiographic) & $50.4 \%$ & $49.8 \%$ & $51.9 \%$ \\
Uveitis & $6.1 \%$ & $4.0 \%$ & $11.1 \%$ \\
SpA- classification & & & \\
Axial & $49.8 \%$ & $55.7 \%$ & $35.6 \%$ \\
Axial \& peripheral & $50.2 \%$ & $44.3 \%$ & $64.4 \%$ \\
\hline
\end{tabular}

Acknowledgments: ArLAR 2018 Scientific committee for initiating SpA special interest group meeting

Disclosure of Interests: Jamal Al-Saleh Grant/research support from: Novartis, AbbVie, Majid Philippe Abi Saab: None declared, Ahmed Negm Speakers bureau: El-lilly, Farida Balushi: None declared, Rajaie Namas: None declared, Nelly Ziade Speakers bureau: Abbvie, Janssen, Lilly, Novartis, Pfizer, Roche, Sanofi

DOI: 10.1136/annrheumdis-2020-eular.2493

\begin{tabular}{|l|}
\hline AB0667 \\
ACHILLES PAIN PERSISTENCE IN PATIENTS \\
AFFECTED BY SPONDYLOARTHRITIS: \\
ULTRASONOGRAPHIC AND BIOMECHANICAL \\
STUDY.
\end{tabular}

A. Batticciotto ${ }^{1}$, S. Olivieri ${ }^{2}$, R. Talotta ${ }^{3}$, A. Cappelli ${ }^{1}$, A. Preda ${ }^{2}$, P. Sarzi-Puttini ${ }^{4}$. ${ }^{1}$ Rheumatology Unit - Ospedale Di Circolo - Fondazione Macchi - Asst Settelaghi, Varese, Italy; ${ }^{2} I R C C S$ Galeazzi Orthopedic Institute, University of Milan, Milan, Italy; ${ }^{3}$ University of Messina, Messina, Italy; ${ }^{4}$ Rheumatology Unit, L. Sacco University Hospital, Milan, Italy 
Background: Enthesis anatomy and biomechanics have a key role in Spondyloarthritis (SpA) pathogenesis (1) but few data are available about the influence of structural and biomechanical changes of Achilles tendon (AT) on persisting pain in longstanding SpA patients.

Objectives: To correlate AT pain in longstanding SpA patients with ultrasonographic detectable disorders and biomechanical abnormalities.

Methods: We performed a monocentric cross-sectional analysis including 35 consecutive patients affected by SpA (13 with Psoriatic Arthritis, 9 with Enteropathic SpA, 6 with Ankylosing Spondylitis and 7 with Undifferentiated $\mathrm{SpA}$ ) under treatment with anti- TNF agents. A rheumatologic clinical and clinimetric evaluation (AT VAS pain, BASDAI, BASFI, HAQ), an ultrasound study of AT according to the Madrid Sonographic Enthesis Index (MASEI) score and a podiatrist biomechanical evaluation [Foot posture index (FPI), degree of ankle dorsiflexion with the knee extended and flexed] were performed.

Results: Study population (13 F; $22 \mathrm{M}$; mean age $54.9 \pm 13.9$ years; mean disease duration $9.5 \pm 5.0$ years; mean BMI $25.8 \pm 4.4$ ) showed a mean AT VAS pain of $3.4 \pm 2.2$, a mean HAQ of $0.6 \pm 0.6$, a mean BASDAI of $3.3 \pm 2.1$ and a mean BASFI of $2.2 \pm 1.9$. At the ultrasonographic evaluation $47 \%(33 / 70)$ of the AT entheses analysed presented a dishomogeneous echostructure, $31 \%$ $(22 / 70)$ structural thickness, $53 \%(37 / 70)$ calcifications, $10 \%(7 / 70)$ erosions, $44 \%(31 / 70)$ a retrocalcanear bursitis. A power Doppler positivity was found only in $0.07 \%(5 / 70)$ of the AT

At the biomechanical evaluation $50 \%(35 / 70)$ of the feet showed a FPI score between 0 and +5 (neutral foot), 46\% (32/70) a FPI score between +6 and +9 (slight foot pronation) and 6\% (4/70) a FPI score between -1 and -4 (slight foot supination).

The mean degree of ankle dorsiflexion with extended knee was $8.4 \pm 3.9$ with the $61 \%(43 / 70)$ of the patients with a maximum dorsiflexion $<10^{\circ}$ of whom $46 \%$ (20/43) do not recover after the knee flexion.

We found a between the mean degree of left ankle dorsiflexion with extended/ flexed knee both with ultrasound-revealed left AT enthesis calcifications $(p=0.014 / 0.037)$ and with left AT enthesis thickness $(p=0.049 / 0.035)$, and a significant association between the mean degree of right ankle dorsiflexion and extended/flexed knee and ultrasound-revealed right AT calcifications $(p=0.008 / 0.012)$. Moreover, we noticed an inverse correlation between the mean overall degree of ankle dorsiflexion with extended/flexed knee and the BASFI values $(p=0.007 / 0.004)$. AT VAS pain was statistically related with Achilles PDUS signal persistence $(p=0.048)$ but not with US signs of chronic entesopathy or biomechanical alterations [calcification $(p=0.39)$, erosions $(p=0.74)$ ]. The limits of the study were the low number of patients recruited and the lack of a control group.

Conclusion: In this monocentric study on a cohort of SpA patients, we demonstrated a statistically significant correlation between ankle-subtalar joint complex biomechanics alterations, ultrasonographic signs of chronic enthesopathy and clinimetric index of functional disability. Residual Achilles pain seems to be related to US signs of active enthesitis.

References:

[1] The enthesis organ concept and its relevance to the spondyloarthropathies. Benjamin, M and McGonagle, D. s.I.: Adv Exp Med Biol, 2009, Vol. 649.

[2] The Synovio-entheseal Complex and Its Role in Tendon and Capsular Associated Inflammation. McGonagle, E D, Aydin, SZ and Tan, AL. 0, s.I.: J Rheumatol Suppl, 2012, Vol. 89

Disclosure of Interests: None declared

DOI: 10.1136/annrheumdis-2020-eular.5891

\section{AB0668 CLINICAL CHARACTERISTICS OF RADIOGRAPHIC AND NON RADIOGRAPHIC AXIAL SPONDYLOARTHRITIS IN A GROUP OF TUNISIAN SPONDYLOARTHRITIS}

K. Ben Abdelghani ${ }^{1}$, Y. Gzam ${ }^{1}$, A. Fazaa ${ }^{1}$, S. Miladi ${ }^{1}$, K. Ouenniche ${ }^{1}$, L. Souabni ${ }^{1}$, S. Kassab ${ }^{1}$, S. Chekili ${ }^{1}$, A. Laatar ${ }^{1} .{ }^{1}$ Mongi Slim Hospital, Rheumatology, Tunis, Tunisia

Background: In the literature, non radiographic axial Spondyloarthritis (nr-SpA) is predominantly female with a shorter period of evolution and similar peripheral manifestations to radiographic axial spondyloarthritis ( $r-S p A)$. However, we do not have Tunisian studies comparing the two groups of axial spondyloarthritis (ax-SpA).

Objectives: The aim of this study was to assess the epidemiological and clinical differences between nr-SpA and r-SpA in a group of Tunisian ax-SpA.

Methods: Two hundred patients with ax-SpA (ASAS 2009 criteria) were retrospectively included and classified as $r$-Spa characterized by the presence of radiographic sacroiliitis and $\mathrm{nr}-\mathrm{SpA}$ defined by the presence sacroiliitis only on MRI or HLA B27 antigen with other clinical features. The different demographic and clinical parameters were compared between the nr-SpA and r-SpA groups.

Results: One hundred thirty-eight men and 62 women were included with a sex ratio of 2,2 . The mean age was $43,3 \pm 11,2$ years and the mean period of evolution was $10,7 \pm 8,4$ years. The patients were divided to $r-S p A$ in $80 \%$ of cases ( $n$ $=160)$ and $n r-S p A$ in $20 \%$ of cases $(n=40)$.

Women were more present in the nr-SpA group with $47,5 \%$ of women versus $26.8 \%$ of women in the $r-S p A$ group $(p=0,01)$. The patients with nr-SpA were younger with a mean age of $39,4 \pm 13,4$ years versus $44,3 \pm 10,4$ years in patients with $r-S p A(p=0,03)$. The mean period of evolution was shorter in nr-SpA group $(5,8 \pm 4,9$ years vs $11.9 \pm 8.5 ; p<0.001)$. The family his tory of SpA was more frequent in $\mathrm{nr}-\mathrm{SpA}$ group (17.5\% vs $4.3 \%, \mathrm{p}=0.004)$. Arthritis were more frequent in nr-SpA (42.5\% vs $13.7 \%$; $p<0.0001)$. Similarly, enthesitis were more frequent in nr-SpA group (45\% vs $15.6 \%$; $<0.0001)$.

No statistically significant differences were found in the following parameters: age at onset of symptoms, diagnostic delay, HLA B-27 antigen and dactylitis.

Conclusion: The clinical features were different in the 2 groups of ax-SpA: Patients with nr-SpA were more female and had more peripheral manifestations while patients with $r-S p A$ were older and with longer period of evolution.

References:

[1] de Winter JJ, et al. Arthritis Res Ther 2016; 18:196

[2] Malaviya AN, et al. Int J Rheum Dis 2015; 18(7):736-41

Disclosure of Interests: None declared

DOI: 10.1136/annrheumdis-2020-eular.6041

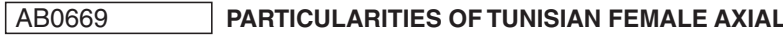 SPONDYLOARTHRITIS}

K. Ben Abdelghani ${ }^{1}$, Y. Gzam ${ }^{1}$, A. Fazaa ${ }^{1}$, S. Miladi ${ }^{1}$, K. Ouenniche ${ }^{1}$,

S. Kassab ${ }^{1}$, L. Souabni ${ }^{1}$, S. Chekili ${ }^{1}$, A. Laatar ${ }^{1} .{ }^{1}$ Mongi Slim Hospital,

Rheumatology, Tunis, Tunisia

Background: Axial spondyloarthritis (ax-SpA) is a chronic rheumatic disease that mainly affects men. However, the female form of ax-SpA remains insufficiently studied.

Objectives: The aim of this study was to determine the clinical characteristics, the disease activity and the functional impact of female ax-SpA in comparison with male ax-SpA.

Methods: This is a retrospective study including patients diagnosed with ax-SpA fulfilling the criteria of the Assessment of SpondyloArthritis international Society (ASAS) 2009

Clinical parameters, erythrocyte sedimentation rate (ESR), C-reactive protein (CRP), Bath ankylosing spondylitis disease activity index (BASDAI) and Bath ankylosing spondylitis functional index (BASFI) were compared between groups of female and male ax-SpA

Results: Two hundred ax-SpA patients were included with $31 \%$ of female $(n=62)$ and a mean age of $43,3 \pm 11,2$ years.

The mean age at onset of symptoms was 31,8 \pm 8,9 years for women and 25,3 \pm 9,1 years for men $(p<0,0001)$. The mean age at diagnosis was $36,4 \pm 9,6$ years for women and $31,7 \pm 10,4$ years for men $(p=0,003)$. Ax-SpA with juvenile onset was noted in $1,7 \%$ of women and $12,1 \%$ of men $(p=0,02)$. Male ax-SpA were significantly more smokers $(46.8 \%$ vs $5.4 \%$; $p<0.001)$. The mean duration of morning stiffness was $11,3 \pm 9,2$ minutes for women versus $21,6 \pm 19,3$ minutes for men $(p=0,005)$

The mean ESR was $42,4 \pm 29,8 \mathrm{~mm}$ for women and $28,3 \pm 23,4 \mathrm{~mm}$ for men ( $p$ $=0,001)$. Radiographic sacroiliitis was present in $69,3 \%$ of women versus $84,7 \%$ of men $(p=0,01)$. The use of anti-TNF alpha was less frequent in women $(29 \%$ vs $48,5 \% ; p=0,01$ )

Our study didn't found a statistically significant difference in peripheral manifestations, extraarticular manifestations, CRP, BASDAI and BASFI between the two groups.

Conclusion: Female ax-SpA seems to have a better prognosis than male with older age in disease onset, less inflammation, less radiographic sacroiliitis and less use of biological treatments.

References:

[1] Rusman T, et al. Curr Rheumatol Rep. 2018; 20(6).

[2] Siar N, et al. Curr Rheumatol Rev. 2019;

Disclosure of Interests: None declared

DOI: 10.1136/annrheumdis-2020-eular.6203 